\title{
Etude de la qualité physico-chimique et biologique des eaux du fleuve Niger à Niamey
}

\author{
Bassirou ALHOU ${ }^{1 *}$, Jean-Claude MICHA ${ }^{2}$, Abdelkader DODO ${ }^{3}$ et \\ Aboubacar AWAISS ${ }^{4}$ \\ ${ }^{1}$ Université Abdou Moumouni de Niamey, Ecole Normale Supérieure, Département Sciences \\ de la Vie et de la Terre, B.P: 10963 Niamey (Niger). Tél: (+227) 969614 22; \\ Fax: (+227)20733072)E-mail:alhoubass@yahoo.fr \\ ${ }^{2}$ FUNDP, Département de biologie, Unité de recherche en biologie des organismes, \\ 61, Rue de Bruxelles B-5,000 Namur (Belgique), E-mail: jean-claude.micha@fundp.ac.be \\ ${ }^{3}$ Université Abdou Moumouni de Niamey, Faculté des sciences, Département de géologie, \\ B.P: 10662 Niamey (Niger), E-mail: dodoabdelkader@yahoo.fr \\ ${ }^{4}$ Université ABDOU Moumouni de Niamey, Faculté d'agronomie, Département des eaux et forêts, \\ BP: 10 960,E-mail: aawaiss_wwf@wwfafrica.org \\ Auteur correspondant, E-mail: alhoubass@yahoo.fr, Tél: (+227) 969614 22, Fax: (+227) 20733072)
}

\section{RESUME}

Le fleuve Niger est le récepteur principal des rejets d'eaux usées de la ville de Niamey, capital du Niger, provenant des activités industrielles, domestiques, hospitalières et agricoles. La quantité des déchets solides produits chaque année est estimée à 273750 tonnes. Or, les eaux du fleuve sont utilisées entre autres pour l'alimentation en eau potable de Niamey et pour l'agriculture irriguée. Les eaux souterraines qui alimentent le fleuve Niger, se dégradent au fil des années au voisinage de Niamey à cause de l'infiltration des eaux issues de la pollution urbaine ainsi que des latrines et fosses sceptiques. La présente étude vise à évaluer la qualité des eaux du fleuve Niger en se basant sur la physico-chimique et les communautés de macroinvertébrés. 36 taxons de macroinvertébrés ont été récoltés le long du fleuve. La description de ces taxons montre une variation de la structure des communautés caractérisée par une baisse de la richesse taxonomique en aval des points de rejet. La présence des taxons comme, Melania sp., les familles de Syrphidae et Culicidae indique une détérioration de la qualité des eaux due à la matière organique dans certains sites proches des points de rejet. En revanche, la présence de Thraulus sp., Elassoneuria sp., Afronurus sp., Centroptiloides sp., Adenophlebia sp., Dipseudopsis sp. et Neoperla sp., reflète une eau de meilleure qualité dans les sites de référence situés en amont de tous les points de rejet. L'analyse canonique des correspondances révèle que, l'oxygène dissous, le phosphore total, les orthophosphates, le $\mathrm{pH}$ et la Demande Chimique en Oxygène $(\mathrm{DCO})$ sont les principaux facteurs qui expliquent de manière significative $(\mathrm{P}<0,05)$ la distribution des taxons le long du fleuve Niger à Niamey.

(C) 2009 International Formulae Group. All rights reserved.

Mots clés: Fleuve Niger, Niamey, qualité de l'eau, macro invertébrés, paramètres physico-chimiques.

\section{INTRODUCTION}

A Niamey, les eaux usées industrielles, domestiques, hospitalières et agricoles sont directement déversées dans le fleuve Niger sans un traitement adéquat (Anonyme, 2001). Tandis que, l'approvisionnement en eau potable de cette ville est assuré par les prélèvements des eaux de ce fleuve opérés en amont. Avec l'urbanisation croissante de la ville, les stations de pompage pour l'approvisionnement de celle-ci sont actuellement situées dans la localité et donc affectées par les eaux usées ainsi que des 
déchets solides. A cette date, Niamey est doté d'un réseau de collecte d'eaux usées qui est en deçà des besoins d'assainissement de la ville. Ce réseau souvent à ciel ouvert est rendu non fonctionnel car transformé en poubelle, créant ainsi, comme dans la plupart des grandes villes africaines, une situation d'hygiène et d'assainissement déplorable à proximité des habitations (Moulay, 2005). Le réseau retient donc une grande quantité des eaux usées destinée à être déversée dans le fleuve. C'est ainsi que dès les premières pluies, le contenu des caniveaux est drainé vers le fleuve affectant par la même occasion la qualité physico-chimique des eaux (Bariweni et Izonfuo, 2001; Plea et al., 2005) et les conditions d'habitat (Nedeau et al., 2003).

La dégradation de la qualité des eaux du fleuve Niger a, dans la plupart des cas, été appréhendée par l'analyse des paramètres physico-chimiques (Nwokedi et al., 1992; Illia, 2007) plutôt que par l'étude de la réponse des communautés biologiques aux variations des conditions environnementales de ce cours d'eau. Pourtant, la qualité de l'eau d'un écosystème aquatique conditionne sa richesse biologique (Ometo et al., 2000; Piscart et al., 2005; Tarr et al., 2005). La qualité de l'eau est généralement corrélée aux variations longitudinales des conditions environnementales causées par l'agriculture, les effluents domestiques ou industriels (Negishi et Richardson, 2003; Roy et al., 2003; Biljana et Sanja, 2008). Plusieurs approches utilisant le changement de la richesse taxonomique et de l'organisation fonctionnelle des espèces aquatiques permettent d'évaluer la qualité des cours d'eau (Evrard et Micha, 1995). Il s'agit entre autres de la richesse spécifique et de la relation entre les assemblages des macroinvertébrés et la qualité de l'eau établie à partir de l'analyse canonique des correspondances (Lepš et Smilauer, 2003; Sarkar et al., 2005).

Le but de cette étude est d'évaluer la qualité des eaux du fleuve Niger le long de la ville de Niamey à l'aide des paramètres physico-chimiques et des macroinvertébrés.

Cette évaluation de la qualité des eaux est fondée sur la caractérisation des communautés de macroinvertébrés en termes de richesse taxonomique et sur l'établissement des relations entre ces communautés et les facteurs environnementaux.

Ainsi, la connaissance des macro invertébrés du fleuve Niger et leur affinité avec les facteurs physico-chimiques permettront aux structures en charge de l'eau à Niamey de disposer d'outils de suivi de la qualité des eaux de ce fleuve.

\section{MATERIELS ET METHODES}

Les macroinvertébrés sont échantillonnés le long du fleuve Niger à Niamey (Figure 1) sous un climat aride caractérisé notamment en moyenne sur une période allant de 1995 à 2005, par une pluviométrie de $517,90 \mathrm{~mm}$ et une température de $29,8{ }^{\circ} \mathrm{C}$. L'évapotranspiration totale annuelle pour la même période est de 2 $802,5 \mathrm{~mm}$.

Le fleuve Niger, par sa longueur de $4200 \mathrm{~km}$, occupe le troisième rang parmi les fleuves africains les plus longs. Il traverse le territoire du Niger sur près de $650 \mathrm{~km}$ et constitue le principal cours d'eau du pays.

Son régime d'écoulement est caractérisé par les hautes eaux $\left(1171 \mathrm{~m}^{3} . \mathrm{s}^{-1} \mathrm{de}\right.$ débit) causées par les quantités de pluies sur la partie haute de son bassin versant (Novembre à février), les basses eaux $\left(123 \mathrm{~m}^{3} \cdot \mathrm{s}^{-1}\right.$ de débit) de mars à juin et une remontée faible des eaux $\left(670 \mathrm{~m}^{3} \mathrm{~S}^{-1}\right.$ de débit) à la suite des pluies locales et les affluents situés en rive droite de juillet à septembre.

Les sites d'échantillonnage d'eau et des macroinvertébrés ont été sélectionnés en fonction des points des rejets d'eaux usées provenant de la ville (figure 1, tableau 1). Le site de TON à Tondibia sert de site de référence; il est situé à l'amont de la ville et est exempt de la pollution urbaine. Il permet ainsi de suivre l'évolution de la variation des indicateurs de la pollution dans la ville de Niamey. Les sources de pollution industrielles et anthropogéniques ont été considérées comme site témoin pour être comparées aux échantillons (Tableau 1).

\section{Echantillonnage des eaux}

Les eaux ont été échantillonnées à une profondeur de $30 \mathrm{~cm}$ en dessous de la surface pour des analyses physico-chimiques (Hunt et Wilson, 1986). L'échantillonnage s'est déroulé selon un intervalle de 10 jours en mars, avril, mai et juin des années 2004 et 


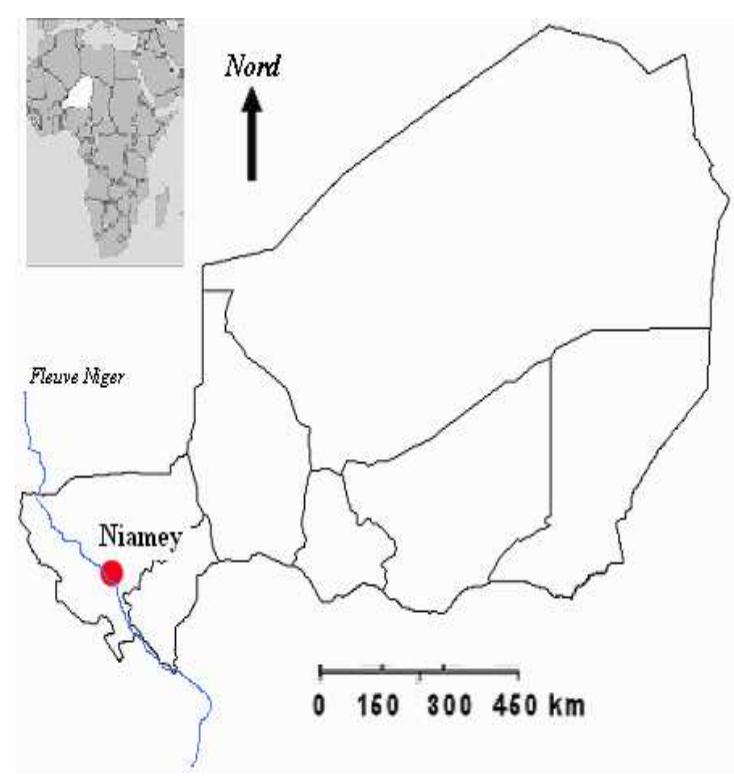

Figure 1a: Localisation de la zone d'étude à l'Ouest du Niger.

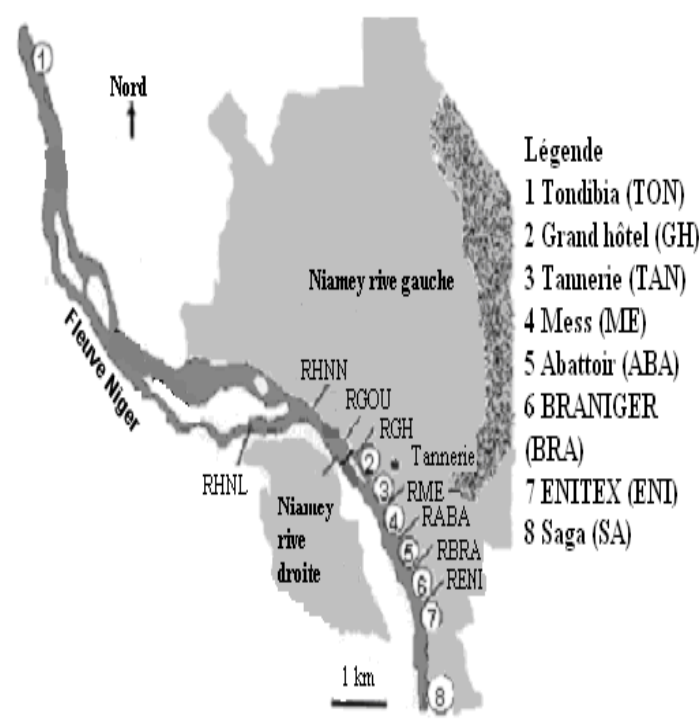

Figure 1b: Carte de localisation des sites d'échantillonnage le long du fleuve Niger à Niamey (zone en gris). (RHNL = Rejets de l'hôpital National de Lamordé; RHNN = Rejets de l'hôpital National de Niamey; RUAM $=$ Rejets de l'Université Abdou Moumouni ; RGOU $=$ Rejets domestiques de Goutiyena ; RBH = Rejets domestiques du Grand hôtel; RME $=$ Rejets domestiques de Mess; RABA $=$ Rejets de l'abattoir; RBRA = Rejets de la brasserie du Niger; RENI $=$ Rejets de 'entreprise Nigérienne de textile).
2005, en juillet, novembre et décembre de l'année 2005, et en janvier, février et mars de l'année 2006. Le période de basses eaux s'étend de mars à juin, celles des hautes eaux de novembre à janvier. La saison des pluies se situe en moyenne entre juillet et septembre

Les échantillons d'eau sont prélevés, dans chaque site, le matin entre 9 et 10 heures, période où le déversement des rejets est le plus important. Ils sont conservés à $4{ }^{\circ} \mathrm{C}$ dans des bouteilles en polyéthylène, propres et stérilisées pour des analyses au laboratoire conformément au manuel de procédure HACH (Isabelle et Claude, 1983).

Les paramètres physico-chimiques (conductivité, $\mathrm{pH}$, température, oxygène dissous) sont immédiatement analysés in situ respectivement avec un conductimètre WTW LF 318/SET (précision $2 \mu \mathrm{s} / \mathrm{cm}$ ); un pH mètre WTW 330i/SET (précision 0,1) et un oxymètre WTW Multiline P3 pH/Oxi-SET (précision $0,1 \mathrm{mg} \mathrm{l}^{-1 \circ} \mathrm{C}$ ).

Les nitrates, nitrites, ammonium, orthophosphates et phosphore total sont analysés au laboratoire respectivement selon la méthode de réduction du cadmium, la méthode de diazotisation, et la méthode de Nessler. Les orthophosphates sont analysés par la méthode phosVer 3 et le phosphore total par la méthode de digestion à l'acide persulfate en utilisant le spectrophotomètre DR/2000. La Demande Chimique en Oxygène (DCO) est dosée 20 jours plus tard par la méthode de digestion au réacteur DCO.

\section{Récolte des macroinvertébrés}

Les macroinvertébrés ont été récoltés en basses eaux (Mai-Juin 2005 et Mars 2006), période la plus adaptée pour l'utilisation d'un filet troubleau de $500 \mu \mathrm{m}$ de maille. Lors de l'échantillonnage, l'ouverture du filet est placée à contre courant et le filet est traîné légèrement sur le fond le long d'un transect de $100 \mathrm{~m}$ de long en passant par le maximum d'habitats possibles (végétation, blocs de pierre). Le filet est ensuite relevé et le contenu bien concentré est reversé dans des bocaux contenant du formol $10 \%$ avant de procéder au tri plus tard au laboratoire. Une seule récolte est organisée par mois et par site le long du littoral.

Les macroinvertébrés sont séparés des substrats en mettant en suspension les organismes par agitation dans l'eau. 
Tableau 1: Sites d'échantillonnage et les sources de pollution associées.

\begin{tabular}{|c|c|c|c|c|}
\hline \multirow[t]{2}{*}{ Sites d'échantillonnage } & \multicolumn{3}{|c|}{ Localisation } & \multirow[t]{2}{*}{ Sources de pollution } \\
\hline & Altitude & Longitude & Latitude & \\
\hline $\begin{array}{l}\text { Tondibia } \\
\text { (TON) }\end{array}$ & 185 & $2^{\circ} 00^{\prime} 33^{\prime \prime} 39 \mathrm{E}$ & $13^{\circ} 33^{\prime} 44^{\prime \prime} 26 \mathrm{~N}$ & $\begin{array}{l}\text { En amont des points } \\
\text { de rejet }\end{array}$ \\
\hline $\begin{array}{l}\text { Grand hotel } \\
(\mathrm{GH})\end{array}$ & 192 & $2^{\circ} 06^{\prime} 54^{\prime \prime} 47 \mathrm{E}$ & $13^{\circ} 29^{\prime} 59^{\prime \prime} 97 \mathrm{~N}$ & $\begin{array}{l}\text { Rejets domestiques } \\
\text { Grand hotel }\end{array}$ \\
\hline Tannerie (TAN) & 175 & $2^{\circ} 06^{\prime} 54^{\prime \prime} 62 \mathrm{E}$ & $13^{\circ} 29^{\prime} 46^{\prime \prime} 86 \mathrm{~N}$ & Tannerie \\
\hline Mess (ME). & 189 & $2^{\circ} 07^{\prime} 07^{\prime \prime} 47 \mathrm{E}$ & $13^{\circ} 29^{\prime} 25^{\prime \prime} 30 \mathrm{~N}$ & Rejets domestiques Mess \\
\hline $\begin{array}{l}\text { Abattoir } \\
\text { (ABA) }\end{array}$ & 195 & $2^{\circ} 07^{\prime} 22^{\prime \prime} 13 \mathrm{E}$ & $13^{\circ} 29^{\prime} 10^{\prime \prime} 12 \mathrm{~N}$ & $\begin{array}{l}\text { Office du Lait du } \\
\text { Niger et abattoir } \\
\text { Office National des }\end{array}$ \\
\hline $\begin{array}{l}\text { BRANIGER } \\
\text { (BRA) }\end{array}$ & 178 & $2^{\circ} 07^{\prime} 38^{\prime \prime} 85 \mathrm{E}$ & $13^{\circ} 28^{\prime} 53^{\prime \prime} 61 \mathrm{~N}$ & $\begin{array}{l}\text { Produits Pharmaceutiques } \\
\text { et Chimiques, BRANIGER, } \\
\text { UNILEVER, Niger lectricité }\end{array}$ \\
\hline $\begin{array}{l}\text { Entreprise Nigérienne } \\
\text { de textile (ENI) }\end{array}$ & 179 & $2^{\circ} 07^{\prime} 46^{\prime \prime} 90 \mathrm{E}$ & $13^{\circ} 28^{\prime} 37^{\prime \prime} 68 \mathrm{~N}$ & $\begin{array}{l}\text { Entreprise Nigérienne } \\
\text { de textile (ENI) }\end{array}$ \\
\hline Saga (SA) & 189 & $2^{\circ} 07^{\prime} 55^{\prime \prime} 49 \mathrm{E}$ & $13^{\circ} 28^{\prime} 05^{\prime \prime} 43 \mathrm{~N}$ & En aval des points de rejet \\
\hline
\end{tabular}

L'échantillon est ensuite passé à travers une colonne de tamis de $5 \mathrm{~mm}, 1 \mathrm{~mm}$ et $0,4 \mathrm{~mm}$ de mailles afin de retirer les organismes. Ces derniers sont identifiés grâce à la clé taxonomique de Durand et Lévêque (1981). Les taxons sont ainsi conservés dans du formol $10 \%$.

\section{Analyses statistiques}

Trois approches sont utilisées pour analyser les données :

i) l'analyse de variance (ANOVA) grâce au package statistica 5.1 (STATSOFT France, 1997) est utilisée sur des données transformées pour tester l'inter-variation des paramètres de qualité des eaux mesurés (Statsoft France, 1997). Le test de Hartlet est utilisé pour vérifier l'homogénéité des variances. Les différences significatives entre les sites sont déterminées en utilisant le test de Scheffé.

ii) l'analyse de la structure des communautés est basée sur la richesse taxonomique, l'indice de diversité de Shannon-Weaver et l'indice d'équitabilité de Shannon (Spellerberg et Fedor, 2003). Les formules utilisées pour calculer ces indices sont les suivantes :

$$
\mathrm{H}=-\sum_{i}^{S}(P i)\left(\log _{2} P i\right)
$$

Avec $\mathrm{H}=$ indice de diversité; $\mathrm{S}=$ nombre de taxons $; \mathrm{Pi}=$ proportion du i ème taxon dans la population.

$$
E=\frac{H}{\log _{2}(S)}
$$

Avec $\mathrm{E}=$ indice d'équitabilité; $\mathrm{H}=$ indice de diversité de Shannon-Weaver et $\mathrm{S}=$ nombre de taxons.

iii) l'Analyse Canonique de Correspondance (ACC) grâce au package CANOCO pour Windows version 4.02 est utilisée pour établir les corrélations significatives entre les taxons et les variables environnementales. Le test de Monte Carlo a permis de sélectionner l'ensemble des facteurs environnementaux qui contribuent de manière significative à la distribution des taxons le long du fleuve Niger à Niamey. Cette méthode permet d'éliminer toutes les variables environnementales qui présentent un facteur d'inflation supérieur à 10 (Ter Braak et Smilauer, 1999). Ces variables corrélées aux autres variables influencent faiblement la distribution des taxons.

Les données sur l'abondance des macroinvertébrés ont subi dans CANOCO for Windows 148 une transformation $\log (\mathrm{x}+1)$ pour normaliser et stabiliser la variance. L'option pondérée par rapport aux taxons rares a été choisie dans le même logiciel.

L'abondance des taxons correspond au nombre total d'individus dénombrés par site et par mois. 
Tableau 2: Caractéristiques physico-chimiques des eaux aux sites d'échantillonnage le long du fleuve Niger à Niamey.

\begin{tabular}{|c|c|c|c|c|c|c|c|c|}
\hline Sites/Paramètres & TON & GH & TAN & ME & ABA & BRA & ENI & $\mathbf{S A}$ \\
\hline \multicolumn{9}{|l|}{$\mathrm{T}\left({ }^{\circ} \mathrm{C}\right)$} \\
\hline M & 25.5 & 26.4 & 25.9 & 26.2 & 26.4 & 26.2 & 21.6 & 25.9 \\
\hline Ec & 3.01 & 3.41 & 3.13 & 3.37 & 3.31 & 3.18 & 3.21 & 3.05 \\
\hline Min & 20 & 20.5 & 20.5 & 20.5 & 21.2 & 21 & 21 & 20.9 \\
\hline Max & 29 & 30.5 & 29.8 & 30.1 & 30.5 & 29.9 & 30.3 & 29.5 \\
\hline \multicolumn{9}{|l|}{$\mathrm{CE}\left(\mu \mathrm{S} \mathrm{cm}^{-1}\right)$} \\
\hline M & 60.6 & 92.7 & 70.2 & 66.6 & 63.8 & 118.2 & 112.8 & 62.9 \\
\hline Ec & 8.82 & 29.18 & 15.10 & 13.18 & 11.03 & 43.38 & 41.76 & 9.98 \\
\hline Min & 46.1 & 47.7 & 48.9 & 46.4 & 46.4 & 48.6 & 46.1 & 46.2 \\
\hline Max & 71.9 & 134.2 & 101.8 & 98.2 & 78 & 169 & 163.6 & 80.3 \\
\hline \multicolumn{9}{|l|}{$\mathrm{pH}$} \\
\hline $\mathrm{M}$ & 6.8 & 7.2 & 6.9 & 7 & 6.6 & 6.7 & 7.8 & 7 \\
\hline Ec & 0.37 & 0.57 & 0.38 & 0.37 & 0.19 & 0.28 & 0.93 & 0.43 \\
\hline Min & 6.1 & 6.1 & 6.2 & 7.8 & 6.4 & 6.2 & & 6.1 \\
\hline Max & 7.3 & 8 & 7.4 & 7.4 & 7 & 7.2 & 9.5 & 7.5 \\
\hline \multicolumn{9}{|l|}{$\mathrm{DCO}\left(\mathrm{mg} \mathrm{l}^{-1}\right)$} \\
\hline $\mathrm{M}$ & 9 & 28.1 & 22.1 & 22.3 & 57.1 & 129.9 & 35.2 & 13.8 \\
\hline Ec & 3.13 & 13.94 & 11.19 & 11.32 & 61.58 & 96.18 & 31.82 & 5.74 \\
\hline Min & 5 & 7.2 & 6.7 & 7.8 & 7.8 & 6.7 & 5.2 & 4.2 \\
\hline Max & 16.2 & 52.2 & 49.7 & 52.5 & 248.3 & 363.3 & 129.7 & 22.8 \\
\hline \multicolumn{9}{|l|}{$\mathrm{NH}_{4}\left(\mathrm{mg} \mathrm{l}^{-1}\right)$} \\
\hline M & 0.3 & 0.9 & 0.6 & 0.6 & 0.6 & 0.7 & 0.4 & 0.5 \\
\hline $\mathrm{Ec}$ & 0.43 & 0.64 & 0.43 & 0.42 & 0.37 & 0.51 & 0.43 & 0.53 \\
\hline Min & 0.1 & 0.3 & 0.3 & 0.3 & 0.3 & 0.2 & 0.1 & 0.1 \\
\hline Max & 1.7 & 2.9 & 2 & 2 & 1.7 & 2.2 & 1.8 & 2.2 \\
\hline \multicolumn{9}{|l|}{$\mathrm{NO}_{3}\left(\mathrm{mg} \mathrm{l}^{-1}\right)$} \\
\hline $\mathrm{M}$ & 0.3 & 0.4 & 0.4 & 0.4 & 0.3 & 0.3 & 0.3 & 0.3 \\
\hline Ec & 0.15 & 0.17 & 0.15 & 0.16 & 0.17 & 0.15 & 0.15 & 0.14 \\
\hline Min & 0.1 & 0.1 & 0.1 & 0.1 & 0.1 & 0 & 0.1 & 0.1 \\
\hline Max & 0.6 & 0.6 & 0.6 & 0.6 & 0.6 & 0.5 & 0.5 & 0.5 \\
\hline \multicolumn{9}{|l|}{$\mathrm{NO}_{2}\left(\mathrm{mg} \mathrm{l}^{-1}\right)$} \\
\hline $\mathrm{M}$ & 0.002 & 0.005 & 0.004 & 0.002 & 0.002 & 0.002 & 0.002 & 0.002 \\
\hline Ec & 0.001 & 0.002 & 0.002 & 0 & 0.001 & 0.001 & 0 & 0 \\
\hline Min & 0.001 & 0.001 & 0.001 & 0.002 & 0.001 & 0.001 & 0.002 & 0.002 \\
\hline Max & 0.003 & 0.007 & 0.011 & 0.003 & 0.003 & 0.003 & 0.003 & 0.003 \\
\hline \multicolumn{9}{|l|}{$\mathrm{PO}_{4}\left(\mathrm{mg} \mathrm{l}^{-1}\right)$} \\
\hline M & 0.1 & 0.2 & 0.2 & 0.2 & 0.2 & 0.2 & 0.1 & 0.1 \\
\hline $\mathrm{Ec}$ & 0.04 & 0.09 & 0.09 & 0.08 & 0.05 & 0.07 & 0.03 & 0.03 \\
\hline Min & 0.1 & 0.1 & 0 & 0.1 & 0.1 & 0.1 & 0.1 & 0 \\
\hline Max & 0.2 & 0.4 & 0.4 & 0.3 & 0.3 & 0.4 & 0.2 & 0.2 \\
\hline \multicolumn{9}{|l|}{$\operatorname{Ptot}\left(\mathrm{mg} \mathrm{l}^{-1}\right)$} \\
\hline M & 0.2 & 0.4 & 0.2 & 0.2 & 0.6 & 0.5 & 0.4 & 0.2 \\
\hline $\mathrm{Ec}$ & 0.13 & 0.39 & 0.12 & 0.22 & 0.84 & 0.51 & 0.49 & 0.22 \\
\hline Min & 0 & 0.1 & 0.1 & 0.1 & 0.1 & 0 & 0 & 0 \\
\hline Max & 0.6 & 1.7 & 0.6 & 0.9 & 3.2 & 2.1 & 1.9 & 0.9 \\
\hline \multicolumn{9}{|l|}{$\mathrm{O}_{2}\left(\mathrm{mg} \mathrm{l}^{-1}\right)$} \\
\hline $\mathrm{M}$ & 7.4 & 7.2 & 6.9 & 7 & 6.6 & 5.6 & 6.5 & 7 \\
\hline $\mathrm{Ec}$ & 0.29 & 1.09 & 0.60 & 0.37 & 0.72 & 0.49 & 0.64 & 0.51 \\
\hline Min & 7.1 & 3.8 & 5.3 & 6.2 & 4.7 & 4.1 & 4.7 & 6.1 \\
\hline Max & 8.2 & 8.2 & 7.6 & 7.8 & 7.5 & 6 & 7.4 & 7.9 \\
\hline
\end{tabular}

"N = 7" (TON=Tondibia, GH=Grand Hôtel, TAN=Tannerie, ME=Mess, ABA=Abattoir, BRA=BRANIGER, ENI= Entreprise Nigérienne de textile, $\mathrm{SA}=\mathrm{Saga}, \mathrm{T}=$ température; $\mathrm{CE}=$ conductivité électrique; $\mathrm{DCO}=$ demande chimique en oxygène; $\mathrm{P}_{\mathrm{tot}}=$ phosphore total; $\mathrm{O}_{2}=$ oxygène dissous; $\mathrm{M}=$ moyenne; $\mathrm{Ec}=\mathrm{Ecartype}, \mathrm{Min}=$ minimum, $\mathrm{Max}=$ maximum). 


\section{RESULTATS}

Analyse des facteurs environnementaux Les résultats d'analyse des caractéristiques physico-chimiques de la qualité des eaux du fleuve Niger à Niamey sont regroupés dans le tableau 2.

Les paramètres tels que $\mathrm{DCO}, \mathrm{O}_{2}$ dissous, $\mathrm{NH}_{4}, \mathrm{PO}_{4}$, Ptot, $\mathrm{pH}$, et $\mathrm{CE}$ montrent des différences significatives $(\mathrm{P}<0,05)$ entre le site TON et certains sites situés en aval immédiat des points de rejet (GH, TAN, ME, ABA, BRA, ENI).

Les valeurs en DCO varient de 4 à 363 $\mathrm{mg} \mathrm{l}^{-1}$, celles de $\mathrm{O}_{2}$ dissous de 4 à $8 \mathrm{mg} \mathrm{l}^{-1}$,

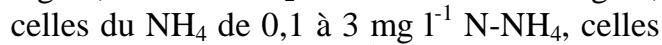
du $\mathrm{PO}_{4}$, de 0 à $0,4 \mathrm{mg} \mathrm{l}^{-1}$, celles du Ptot, 0 à 3,2 mg. $\mathrm{l}^{-1} \mathrm{P}$, celles du pH 6 à 9,5, et celles du $\mathrm{CE}$ de 46 à $164 \mu \mathrm{S} \mathrm{cm}^{-1}$.

\section{Identification des macroinvertébrés}

Au total 43122 individus appartenant à 36 taxons (insectes $80 \%$, mollusques $14 \%$ ) ont été récoltés (tableau 3). En termes d'abondance totale, les Thiaridae $(89 \%)$ et les Chironomidae $(8 \%)$ sont numériquement les plus dominants.

Selon les sites, les groupes taxonomiques observés diffèrent. Ainsi, les Ephéméroptères (Ephemerellina sp., Centroptiloides sp., Afronurus sp., Thraulus sp., Elassoneuria sp., Caenis sp., Cloen sp.), Plécoptères (Neoperla sp.), Odonates (Pseudagrion sp.), Trichoptères (Dipseudopsis sp., Macronema sp., Ecnomus sp.), Coléoptères (Enochrus sp., Hydrochus sp.) et les Crustacées (Potamonautes sp.) sont principalement récoltés en amont (au site de référence) et en aval de tous les points de rejet (au site $\mathrm{SA}$ ).

En revanche, les Diptères (Chironomidae, Syrphidae, Tabanidae, Ceratopogonidae), les Mollusques (Pila sp., Aspatharia sp., Lymnaea sp., Melania sp.), les Coléoptères (Neohydrophilus sp., Canthydrus sp., Rhantaticus sp.), les Odonates (Libellulidae, Ictinogomphus ferax, Phyllogomphus sp.), les Hétéroptères (Anisops sp., Naucoris sp., Hydrocyrius sp., Micronecta sp.) et les Crustacées (Caridina sp.) sont présents en aval immédiat des points de rejet.

Quant à l'indice de diversité de Shannon-Weaver, il décroît significativement $(\mathrm{P}<0,05)$ en passant de l'amont à l'aval immédiat des points de rejet (figure 2). Il est inférieur à 1 à TAN et supérieur ou égal à 4 à TON. L'analyse de l'indice d'équitabilité de Shannon montre la même évolution que l'indice de diversité de Shannon-Weaver (0,63 à TON et 0 à TAN), (figure 3 ).

Corrélation entre les macroinvertébrés et les paramètres physico-chimiques

Les deux premiers axes de l'ACC expliquent $48 \%$ de variance (figure 4). L'inertie totale expliquée par les deux axes est de 1,793. La part d'inertie significativement expliquée par les facteurs environnementaux représente $64 \%$ soit 1,146 . Ces facteurs sont la profondeur de l'eau aux sites d'échantillonnage, l'oxygène dissous, le phosphore total, les orthophosphates et le $\mathrm{pH}$. Le premier axe est positivement corrélé à la profondeur tandis que le second axe l'est à l'oxygène dissous et au phosphore total.

191 Quant aux espèces, Melania sp., Lymnaea sp., Aspatharia sp., Caridina sp. et Phyllogomphus sp. le sont à l'axe 1 alors que Potamonautes sp., Centroptilö̈des sp., Elassoneuria sp., Ephemerellina sp., Pseudagrion sp., Hydrochus sp., Ecnomus sp. et Macronema sp. sont négativement corrélées à cet axe (figure 4a). Par ailleurs, les Nématodes, Pila sp., Libellulidae, Micronecta sp., Ceratopogonidae et Chironomidae sont positivement corrélées à l'axe 2 et Sphaerium sp., Thraulus sp., Caenis sp. et Enochrus sp. sont négativement corrélées à cet axe.

L'analyse de la figure $4 \mathrm{~b}$ montre la différence entre les sites.

\section{DISCUSSION}

Du point de vue physico-chimique, les concentrations en $\mathrm{NH}_{4}$ du fleuve Niger sont significativement $(\mathrm{P}<0,5)$ plus élevées que celles des nitrates; ce qui peut s'expliquer par un apport en matière organique favorable au processus de l'ammonification au détriment de la nitrification (Bartsch et Ingram, 1967). Dans l'ensemble, les concentrations en nitrates sont faibles $\left(<0,5 \quad \mathrm{mg} . \mathrm{l}^{-1}\right)$ et comparables à celles enregistrées par Uzoukwu et al. (2004) dans la rivière de Ubu dans le delta du Niger au Nigeria $(0,1$ à 0,3 mg. $1^{-1} \mathrm{NO}^{-3}$ ).

A certaines stations $(\mathrm{GH}, \mathrm{ABA}, \mathrm{BRA}$, $\mathrm{ENI}$ ), les concentrations en oxygène dissous sont souvent en dessous de $5 \mathrm{mg} \mathrm{l}^{-1}$, teneur requise pour la survie des êtres vivants 
Tableau 3: Abondance totale des macroinvertébrés récoltés aux différents sites d'échantillonnage le long du fleuve Niger à Niamey.

\begin{tabular}{|c|c|c|c|c|c|c|c|c|}
\hline \multirow[b]{2}{*}{ Taxons } & \multicolumn{8}{|c|}{ Sites } \\
\hline & TON & GH & TAN & ME & $\mathbf{A B A}$ & BRA & ENI & SA \\
\hline Nématodes & 26 & 67 & & 3 & 1 & 13 & 1 & \\
\hline \multicolumn{9}{|l|}{ Mollusques } \\
\hline \multicolumn{9}{|l|}{ Gastéropodes } \\
\hline \multicolumn{9}{|l|}{ Pilidae } \\
\hline Pila sp. (Röding) & & 11 & & & 1 & & & \\
\hline \multicolumn{9}{|l|}{ Thiaridae } \\
\hline Melania sp. (Olivier) & 1 & 49 & 37917 & 443 & 120 & 1 & 1 & 1 \\
\hline \multicolumn{9}{|l|}{ Lymnaeidae } \\
\hline Lymnaea sp. (Lamarck) & 5 & & 13 & 5 & 68 & 2 & & 4 \\
\hline \multicolumn{9}{|l|}{ Bivalvia } \\
\hline \multicolumn{9}{|l|}{ Sphaeridae } \\
\hline Sphaerium sp. (Scopoli) & 26 & & & & 25 & 16 & & 26 \\
\hline \multicolumn{9}{|l|}{ Mutelidae } \\
\hline Aspatharia sp. (Bourguignat) & 1 & 3 & 7 & 10 & 37 & 36 & 1 & 13 \\
\hline \multicolumn{9}{|l|}{ Crustacées } \\
\hline \multicolumn{9}{|l|}{ Atyidae } \\
\hline Caridina sp. (Kingsley) & 3 & & 20 & 5 & 23 & & & \\
\hline \multicolumn{9}{|l|}{ Potamidae } \\
\hline Potamonautes sp. (Macleay) & 3 & & & & & & & \\
\hline \multicolumn{9}{|l|}{ Ephéméroptères } \\
\hline \multicolumn{9}{|l|}{ Leptophlebiidae } \\
\hline Thraulus sp. (Eaton) & 58 & & & & 7 & 3 & & 163 \\
\hline \multicolumn{9}{|l|}{ Caenidae } \\
\hline Caenis sp. (Stephens) & 50 & & & & 66 & & & 127 \\
\hline \multicolumn{9}{|l|}{ Baetidae } \\
\hline Centroptiloides sp. (Lestage) & 6 & & & & & & & \\
\hline Cloen sp. & 2 & & & & & & & \\
\hline \multicolumn{9}{|l|}{ Heptageniidae } \\
\hline Afronurus sp. (Lestage) & 1 & & & & & & & \\
\hline \multicolumn{9}{|l|}{ Oligoneuriidae } \\
\hline Elassoneuria sp. (Eaton) & 7 & & & & & & & 5 \\
\hline \multicolumn{9}{|l|}{ Ephemerellidae } \\
\hline Ephemerellina sp.(Lestage) & 3 & & & & & & & \\
\hline \multicolumn{9}{|l|}{ Odonates } \\
\hline \multicolumn{9}{|l|}{ Gomphidae } \\
\hline Ictinogomphus ferox (Rambur) & & 3 & & & & & & \\
\hline
\end{tabular}




\begin{tabular}{|c|c|c|c|c|c|c|c|c|}
\hline Phyllogomphus sp. (Selys) & 6 & 3 & 15 & 16 & 1 & 2 & 3 & \\
\hline \multicolumn{9}{|l|}{ Coenagriidae } \\
\hline Pseudagrion sp. (Selys) & 18 & & & & & & & 7 \\
\hline Libellulidae & 4 & 31 & 1 & & 1 & & 1 & 7 \\
\hline \multicolumn{9}{|l|}{ Hétéroptères } \\
\hline \multicolumn{9}{|l|}{ Corixidae } \\
\hline Micronecta sp. (Hutch) & 5 & 6 & & & & & 18 & \\
\hline \multicolumn{9}{|l|}{ Naucoridae } \\
\hline Naucoris sp. (Geoffroy) & & & & 2 & & & & \\
\hline \multicolumn{9}{|l|}{ Notonectidae } \\
\hline Anisops sp. (Spinola) & & & & & 1 & & 1 & \\
\hline \multicolumn{9}{|l|}{ Belostomidae } \\
\hline Hydrocyrius columbiae & & & & 3 & & & & \\
\hline \multicolumn{9}{|l|}{ Plécoptères } \\
\hline \multicolumn{9}{|l|}{ Perlidae } \\
\hline Neoperla sp. (Needham) & 1 & & & & & & & \\
\hline \multicolumn{9}{|l|}{ Coléoptères } \\
\hline \multicolumn{9}{|l|}{ Dytiscidae } \\
\hline Rhantaticus sp. (Sharp) & & & & & & & & 1 \\
\hline Canthydrus sp. (Sharp) & & & 1 & 2 & & 2 & & 5 \\
\hline \multicolumn{9}{|l|}{ Hydrochidae } \\
\hline Hydrochus sp. (Leach) & & & & & & & & 5 \\
\hline \multicolumn{9}{|l|}{ Hydrophilidae } \\
\hline Neohydrophlilus sp. (Orchymont) & & 2 & 2 & & & 3 & & 1 \\
\hline Enochrus sp. (Thomson) & & & & & & & & 2 \\
\hline
\end{tabular}

Trichoptères

Polycentropodidae

Dipseudopsis sp. (Walker) 3

Ecnomidae

Ecnomus sp. (Mac Lachlan)

Hydropsychidae

Macronema sp. (Pictet) 4

Diptères

Syrphidae

Tabanidae

Ceratopogonidae

Chironomidae

Abondance totale

Total taxons (TON=Tondibia, GH=Grand Hôtel, TAN=Tannerie, ME=Mess, ABA=Abattoir, BRA=BRANIGER, ENI= Entreprise Nigérienne de textile, SA=Saga). 

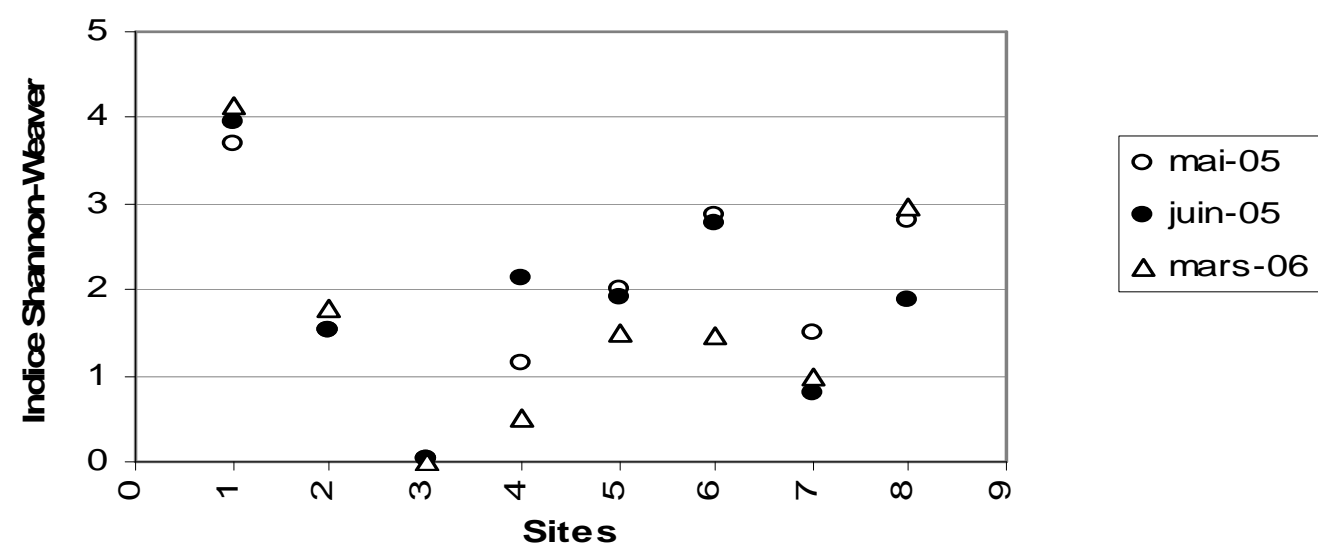

Figure 2: Indice de diversité de Shannon-Weaver. (TON=Tondibia, GH=Grand Hôtel, TAN=Tannerie, ME=Mess, ABA=Abattoir, BRA=BRANIGER, ENI= Entreprise Nigérienne de textile, SA=Saga).
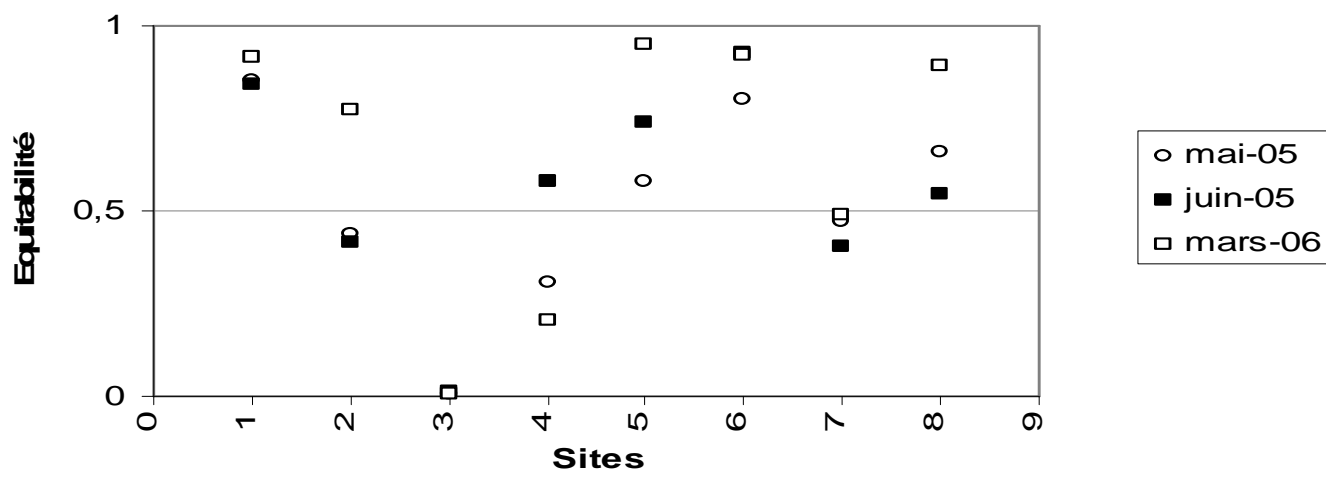

Figure 3: Indice d'équitabilité de Shannon. (TON=Tondibia, GH=Grand Hôtel, TAN=Tannerie, ME=Mess, $\mathrm{ABA}=$ Abattoir, $\mathrm{BRA}=\mathrm{BRANIGER}, \mathrm{ENI}=$ Entreprise Nigérienne de textile, $\mathrm{SA}=$ Saga).

aquatiques (Hodges, 1973; Odokuma et Okpokwasili, 1993; Izonfuo et Bariweni, 2001).

Quant aux valeurs de $\mathrm{pH}$, elles se situent autour de 7 à Niamey et reflètent bien la lithologie à l'échelle du bassin et le faciès bicarbonaté sodique des eaux du fleuve Niger à Niamey et dans le delta supérieur au Mali (Picouet, 1999). La seule hausse importante de $\mathrm{pH}$ relevée à l'aval du rejet de l'ENITEX (RENI) est due à la soude caustique que renferment ces rejets; ce qui leur confère un $\mathrm{pH}$ basique. Il faut noter qu'une forte augmentation de $\mathrm{pH}$ dans l'eau peut être toxique pour la faune aquatique en induisant une augmentation des concentrations en ammoniac toxic (Coimbra et al., 1996;
Department of water affairs and forestry, 1996).

Les eaux du fleuve Niger à Niamey présentent dans l'ensemble une faible minéralisation. Elles présentent également une conductivité proche de celles signalées dans le Niger supérieur au Mali (19 à $40 \mu \mathrm{S} \mathrm{cm}^{-1}$ à Koulikoro) par Picouet (1999) et dans le delta du Niger au Nigeria (48 à $90 \mu \mathrm{S} \mathrm{cm}^{-1}$ à Epie Creek) par Izonfuo et Bariweni (2001).

Par ailleurs, l'analyse des communautés de macroinvertébrés fait ressortir que la plupart des taxons récoltés en amont des points de rejet présentent une faible abondance et une équitabilité supérieure à 0,5 contrairement aux sites situés en aval immédiat des points de rejet où le nombre de 

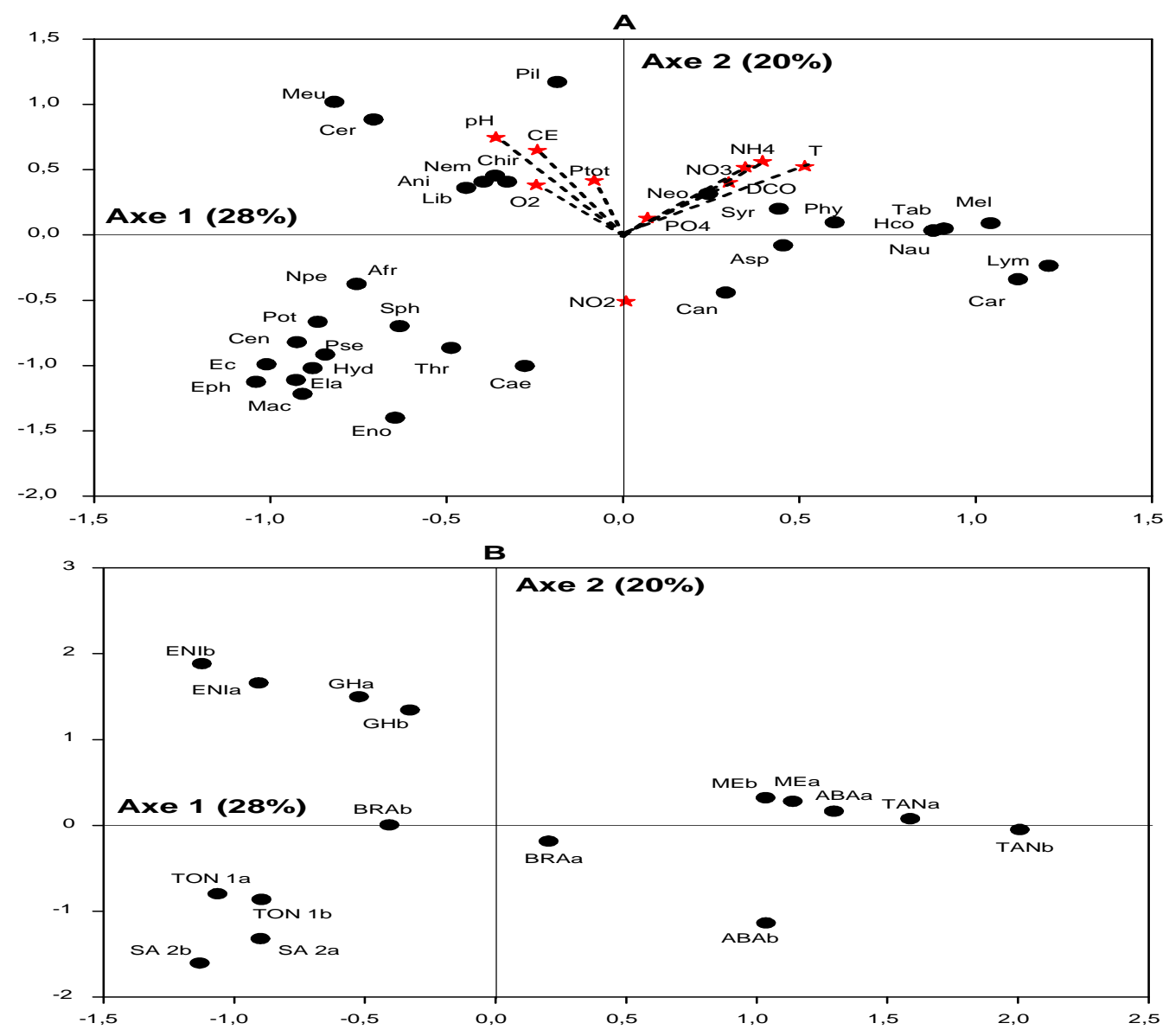

Figure 4: Ordination des facteurs environnementaux et des taxons (A) et des sites d'échantillonnage (B) dans les deux premiers axes de l'ACC. (T=température; $\mathrm{CE}=$ conductivité électrique; $\mathrm{DCO}=$ demande chimique en oxygène; Ptot=phosphore total; $\mathrm{O}_{2}=$ oxygène dissous; $\mathrm{Pr}=$ profondeur, $\mathrm{NO}_{3}=$ nitrates, $\mathrm{NO}_{2}=$ nitrites, $\mathrm{NH}_{4}=$ ammonium, TON=Tondibia, GH=Grand Hôtel, TAN=Tannerie, ME=Mess, ABA=Abattoir, BRA=BRANIGER, ENI= Entreprise Nigérienne de textile, SA=Saga, a = Mai 2005, b = Juin 2005, Afr=Afronurus, Ani=Anisops, Asp=Aspatharia, Cae=Caenis, Can=Canthydrus, Car=Caridina, Cen=Centroptiloides, Cer=Ceratopogonidae, chir=Chironomidae, Ec=Ecnomus, Ela=Elassoneuria, Eno=Enochrus, Eph=Ephemerellina, Hco=Hydrocyrius, Hyd=Hydrochus, Lib=Libellulidae, Lym=Lymnaea, Mac=Macronema, Mel=Melania, Meu=Micronecta, Nau=Naucoris, Nem=Nemathelmintes, Neo=Neohydrophlilus, Npe=Neoperla, Phy=Phyllogomphus, Pil=Pila, Pot=Potamonautes, Pse=Pseudagrion, $\mathrm{Sph}=$ Sphaerium, Syr=Syrphidae, Tab=Tabanidae, Thr=Thraulus). Les facteurs environnementaux actifs sont reliés à l'origine de l'axe par un trait en pointillé.

taxons est faible, l'abondance élevée et l'équitabilité inférieure à 0,5 . Ainsi, dans des conditions de milieu défavorables, les taxons les plus tolérants prolifèrent pendant que les moins tolérants régressent. Cependant, dans des conditions favorables, un équilibre s'établit plus ou moins entre les taxons les plus tolérants et les moins tolérants : c'est le schéma généralement admis en cas d'une altération (Odum, 1972). Ainsi, l'analyse de l'abondance taxonomique montre particulièrement une forte dominance de l'espèce
Melania sp. au site TAN (Figure 5). La présence à ce site d'importants résidus d'Acacia nilotica utilisés dans le tannage des peaux pourrait expliquer la dominance de cette espèce à TAN et dans le site le plus proche de TAN (ME).

A l'abattoir par contre, malgré l'accumulation d'importants contenus stomacaux au fond du site $\mathrm{ABA}$, l'espèce Melania sp. domine avec une faible abondance comparativement à TAN. La proximité de ce site des rejets acides $(\mathrm{pH}$ 

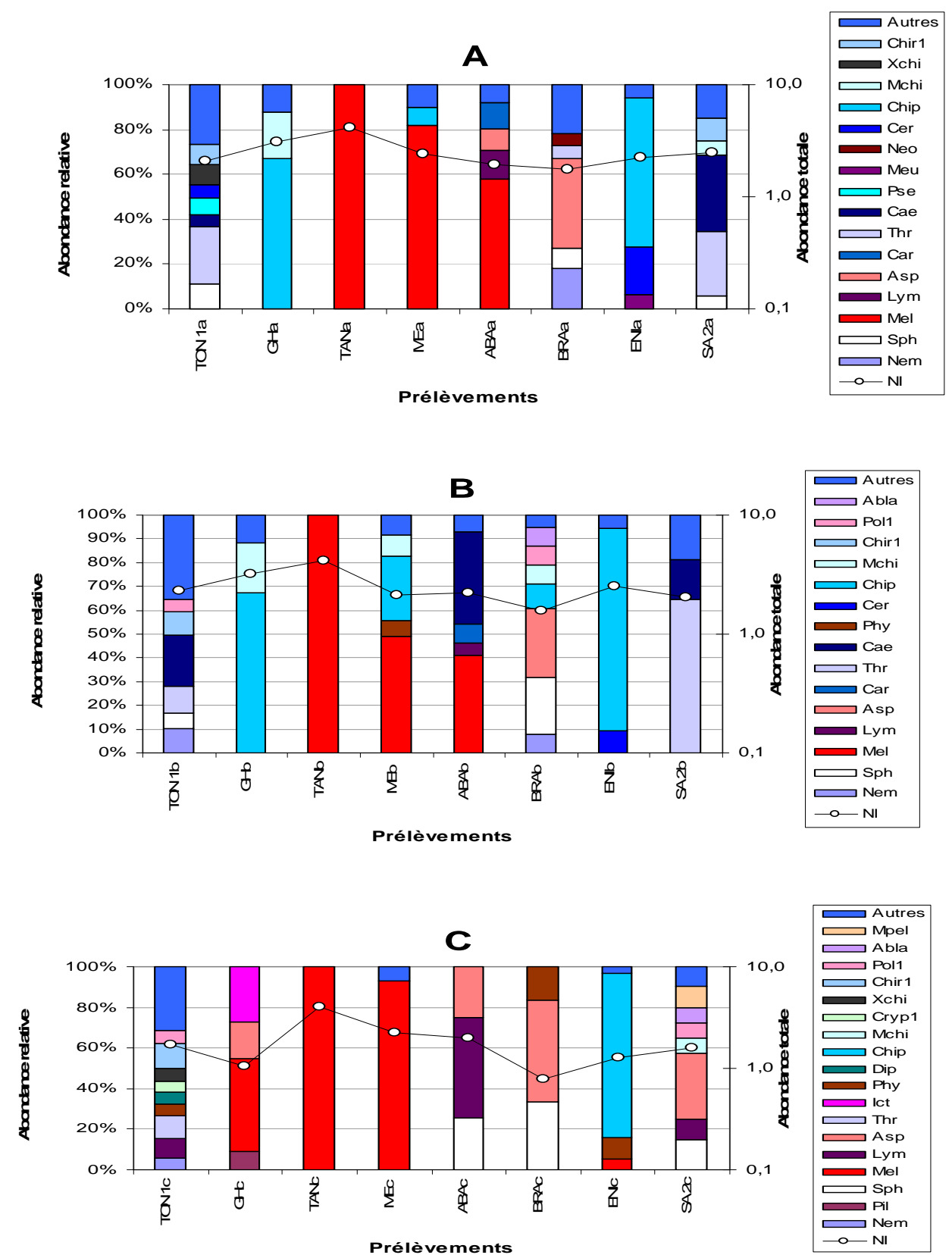

Figure 5: Distribution des abondances taxonomiques totales et relatives dans les prélèvements effectués au filet troubleau en mai 2005 (A), juin 2005 (B) et mars 2006 (C). (Ade =Adenophlebia, Afr = Afronurus, $\mathrm{Agr}=$ Agraptocorixa, $\mathrm{Amp}=$ Amphiops, $\mathrm{Asp}=$ Aspatharia, $\mathrm{Bae}=$ Baetis, $\mathrm{Bio}=\mathrm{Biomphalaria}, \mathrm{Bul}=$ Bulinus, Bur $=$ Burnupia, $\mathrm{Cae}=$ Caenis, Can $=$ Canthydrus, $\mathrm{Car}=$ Caridina, $\mathrm{Cen}=$ Centroptiloides, $\mathrm{Cer}=$ Ceratopogonidae, $\mathrm{Clo}=$ Cloen, Cor = Cordulidae, Dip = Dipseudopsis, Ec = Ecnomus, Ela = Elassoneuria, $\mathrm{ENO}=$ Enochrus, $\mathrm{Eph}=$ Ephemerellina, Hco $=$ Hydrocyrius, $\mathrm{Hel}=$ Helminthocaris, Hir $1=$ Hirudinés 1 , Hir $2=$ Hirudinés 2 , Hir $3=$ Hirudinés 3 , Hme $=$ Hydrometra, Hyd $=$ Hydrochus, Ict = Ictinogomphus, Isc $=$ Ischnura, Lan = Lanistes, Lib $=$ Libellulidae, Lym $=$ Lymnaea, Mac = Macronema, Man = Mansonia, Mel = Melania, Meso = Mesovelia, Meu = Micronecta, Nau $=$ Naucoris, Nem $=$ Nemathelmintes, $\mathrm{Neo}=$ Neohydrophlilus, Not $=$ Notonecta, Npe $=$ Neoperla, Phy $=$ Phyllogomphus, Pil $=$ Pila, Pot $=$ Potamonautes, Pse = Pseudagrion, Ran $=$ Ranatra, Rhan $=$ Rhantaticus, Spe $=$ Spercheus, $\mathrm{Sph}=$ Sphaerium, $\mathrm{Ste}=$ Stenelmis, Syr $=$ Syrphidae, Tab $=$ Tabanidae, $\mathrm{Thr}=$ Thraulus, Tip $=$ Tipulidae $).$ 
moyen = 5) de l'Office du Lait du Niger (OLANI) pourrait être défavorable à l'extension et à la prolifération de cette espèce (Coimbra et al., 1996; Department of Water Affairs and Forestry, 1996; Piyankarage et al., 2004) malgré que les rejets de l'abattoir présentent un $\mathrm{pH}$ neutre compris entre 6 et 7 .

La comparaison des sites basée sur la richesse taxonomique et l'indice de diversité de Shannon-Weaver montre également des différences entre les sites en passant de l'amont à l'aval des points de rejet. Ainsi, la richesse taxonomique baisse de manière significative en aval des points de rejet comparativement au site de référence. Plus en aval, au site SA, une situation de restauration de la diversité commence à s'observer, traduisant ainsi un gradient d'altération en passant de l'amont à l'aval des sources de pollution.

Ces résultats sont en corrélation avec les concentrations en DCO, $\mathrm{O}_{2}, \mathrm{NH}_{4}, \mathrm{PO}_{4}, \mathrm{P}_{\text {tot }}$, $\mathrm{pH}$ et $\mathrm{CE}$ significativement différentes $(\mathrm{P}<0,05)$ entre le site de référence $(\mathrm{TON})$ et certains sites situés en aval immédiat des points de rejet $(\mathrm{GH}, \mathrm{TAN}, \mathrm{ME}, \mathrm{ABA}, \mathrm{BRA}$, ENI).

En effet, la corrélation établie entre les taxons et les paramètres physico-chimiques à partir de l'analyse canonique des correspondances montre un gradient écologique, dans la distribution des taxons, significativement $\quad(\mathrm{P}<0,05)$ expliqué par l'oxygène dissous, le $\mathrm{pH}$, la DCO, les orthophosphates et le phosphore total. Corrélativement à ce gradient écologique, deux groupes taxonomiques se distinguent :

- le premier groupe composé de Thraulus sp., Caenis sp., Centroptilö̈des sp., Baetis sp., Afronurus sp., Ephemerellina sp., Rhantaticus sp., Canthydrus sp. et Ecnomus sp. est négativement corrélé à la DCO, au phosphore total et aux orthophosphates. Cette corrélation reflète la sensibilité de ces taxons à la pollution organique et phosphorée. Ce groupe de taxons composé d'éphéméroptères, de Coléoptères et de Trichoptères est essentiellement présent à TON et SA, deux sites qui sont relativement moins pollués ;

- le second groupe composé de Bulunus

sp., Micronecta sp., Mesovelia sp., Neohydrophilus sp., Syrphidae, Tipulidae, Tabanidae et Ceratopogonidae présente une affinité avec la matière organique. Cette affinité pourrait justifier leur présence dans les sites $\mathrm{GH}, \mathrm{ABA}$ et $\mathrm{BRA}$ où les paramètres physico-chimiques présentent les concentrations les plus élevées.

De plus, l'analyse de la distribution des taxons en passant de l'amont à l'aval des points de rejet montre que les taxons tels que Ephemerellina sp., Centroptiloüdes sp., Thraulus sp., Afronurus sp., Caenis sp., Elassoneuria sp. et Ecnomus sp. sont absents ou deviennent rares dans les zones de rejet pour réapparaître plus loin en aval dans le site SA.

Dans les sites les plus proches des points de rejet apparaissent des taxons tolérants (Chironomidae, Syrphidae...) dans certaines conditions du milieu telles que des faibles concentrations en oxygène dissous (Ndaruga et al., 2004).

Cette distribution des taxons pourrait être attribuée non seulement à la différence de la qualité physico-chimique des eaux du fleuve mais aussi à la différence d'habitats des différents sites d'échantillonnage. Il faut noter que la diversité d'habitats est plus élevée au site de référence (TON) et dans le site le plus éloigné des points de rejet qui est SA. Dans ces sites on note la présence des blocs de pierre absents dans les autres sites. Quinn et Hickey (1990) relatent l'influence des substrats sur la densité et la richesse taxonomique des invertébrés aquatiques.

Ces résultats corroborent plusieurs études qui montrent que les Ephéméroptères, les Plécoptères et les Trichoptères sont des taxons les plus polluo-sensibles alors que les Diptères (Chironomidae, Syrphidae), les Mollusques et les Odonates sont les taxons les plus tolérants (Miserendino et Pizzolon, 2003; Ndaruga et al., 2004; Piscart et al., 2005).

En effet, beaucoup d'études utilisent comme indicateurs de pollution la proportion des Ephéméroptères, Plécoptères et Trichoptères par rapport aux Chironomidae dans un échantillon (Bispo et al., 2006) à cause de leur polluo-sensibilité (Muli et Mavuti, 2001; Ogbogu, 2001; Callisto et al., 2002).

Par ailleurs, la variabilité taxonomique observée entre les échantillonnages effectués à un mois d'intervalle dans un même site pourrait être attribuée aux fluctuations du niveau d'eau du fleuve Niger à cette période (Statzner et Higler, 1986; Shivoga, 2001; Tarr 
et al., 2005) qui n'est pas favorable à l'installation d'une communauté stable de macroinvertébrés. Cette fluctuation entraîne généralement un changement des caractéristiques physico-chimiques le long du cours d'eau (Uzoukwu, 2004). La présence de l'espèce Ecnomus sp. généralement signalée dans des rivières temporaires (Morais, 1995 ; Coimbra et al., 1996) pourrait traduire cette instabilité du niveau de l'eau du fleuve. Ainsi, Richards et al. (1993) soulignent que le régime hydraulique et le substratum sont des conditions physiques qui affectent fortement la composition, l'abondance et la distribution des communautés de macroinvertébrés. Quant à Death (1995), il conclut que la stabilité d'habitats et les interactions biotiques pourraient être des facteurs importants dans la structuration des communautés de macroinvertébrés dans de nombreuses rivières. Il trouve que les communautés de macroinvertébrés sont beaucoup plus importantes dans les sites stables comparativement aux sites instables.

Il est évident à travers cette étude que les eaux du fleuve Niger à Niamey sont affectées par la matière organique et les nutriments (phosphore, ammonium). Ce changement de la qualité des eaux est également traduit en aval de certains points de rejet par la baisse du taux d'oxygène dissous et l'augmentation du $\mathrm{pH}$. Il se répercute ainsi sur la communauté des macroinvertébrés à travers le changement dans la composition spécifique le long du fleuve.

Cette étude a permis d'apprécier l'impact des eaux usées de la ville de Niamey sur la qualité des eaux du fleuve Niger et d'établir pour la première fois à Niamey le lien entre la qualité de l'eau de ce fleuve et sa richesse taxonomique en macroinvertébrés. Elle renseigne également sur les assemblages de macroinvertébrés et leurs valeurs potentielles dans l'évaluation de la qualité biologique et physico-chimique des eaux du fleuve Niger à Niamey.

\section{REMERCIEMENTS}

Cette recherche a été financée par la Coopération Technique Belge (CTB), la Fondation Internationale pour la Science (FIS) et le Comité Scientifique et Technique de l'Organisation de la Conférence Islamique (COMSTECH), Islamabad, Pakistan.
Les auteurs remercient également Docteur François Darchambeaux pour ses appui-conseils et Monsieur Boubé Sami pour sa contribution dans l'analyse physicochimique des échantillons.

\section{RÉFÉRENCES BIBLIOGRAPHIQUES}

Akpan AW. 2004. The water quality of some tropical freshwater bodies in Uyo (Nigeria) receiving municipal effluents, slaughter-house washings and agricultural land drainage. The Environ., 24: 49-55.

Anonym. 2001. Etude sur l'amélioration de l'assainissement de la ville de Niamey en République du Niger. Rapport final provisoire. Vol. II: Rapport principal. Tokyo Engineering Consultants CO, LTD and Yachiyo Engineering CO, LTD, p. 497.

Bariweni AP, Izonfuo LWA. 2001. The effect of urban runoff water and human activities on some physico-chemical parameters of the Epie Creek in the Niger Delta. Journal Appl. Sc. Environ. Manage., 5: 47-55.

Bartsch AF, Ingram WM. 1967. Stream life and the pollution environnement. Biol. Water Pollut., US Department of Interior, F.W.P.C.A.: 119-127.

Biljana R, Sanja P. 2008. Macroinvertebrate diversity in the karst Jadro river (Croatia). Arch. Biol. Sci. Belgrade, 60: 437-448.

Bispo PC, Oliveira LG, Bini LM, Sousa KG. 2006. Ephemeroptera, Plecoptera and Trichoptera assemblages from riffles in mountain streams of Central Brazil: environmental factors influencing the distribution and abundance of immatures. Braz. J. Biol., 66: 611-622.

Brodersen KP, Pedersen O, Lindegaard C, Hamburger K. 2004. Chironomids (Diptera) and oxy-regulatory capacity: An experimental approach to paleolimnological interpretation. Limnol. Oceanogr., 49: 1549-1559.

Callisto M, Moreno P, Gonçalves JFJr, Leal JJF, Esteves FA. 2002. Diversity and biomass of Chironomidae (Diptera) larvae in an impacted coastal lagoon in Rio De Janeiro, Brazil. Braz. J. Biol., 62: 77-84.

Coimbra CN, Graça MAS, Cortes RM. 1996. The effects of a basic effluent on macroinvertebrate community structure in 
a temporary mediterranean river. Environ. Pollut., 94(3): 301-307.

Department of Water Affairs and Forestry. 1996. South African water quality guidelines: Aquatic ecosystems $\left(1^{\text {st }}\right.$ edn; vol. 7). Department of Water Affairs and Forestry: Pretoria, Republic of South Africa.

Death RG. 1995. Spatial patterns in benthic invertebrate community structure: products of habitat stability or are they habitat specific. Freshw. Biol., 33: 455467.

Durand JR, Lévêque C. 1981. Flore et Faune Aquatiques de l'Afrique SaheloSoudanienne. Tome II, ORSTOM: Paris; $847 \mathrm{p}$.

Evrard M, Micha JC. 1995. Relation entre la diversité du substrat et la diversité faunistique dans un bief belge de la rivière Meuse. Ann. Limnol., 31: 93-103.

Hart RC, Campbell LM, Hecky RE. 2003. Stable isotope analyses and demographic responses counter prospects of planktivory by Caridina (Decapoda: Atyidae) in Lake Victoria. Oecol., 136: 270-278.

Hodges L. 1973. Environmental Pollution. A Survey of Emphasising Physical and Chemical Principles. Holt, Rhinehart and Winston, Inc.: New York.

Hunt DTE, Wilson AL. 1986. The Chemical Analysis of Water: General Principles and Techniques. Royal Society of Chemistry: London.

Isabelle D, Claude D. 1983. Manuel d'Analyse de l'Eau HACH. COFRADIS : France; $443 p$.

Izonfuo LWA, Bariweni AP. 2001. The effect of urban runoff water and human activities on some physico-chemical parameters of the Epie Creek in the Niger delta. Journal of Applied Sciences and Environmental Management, 5: 47-55.

Lepš J, Šmilauer P. 2003. Multivariate Analysis of Ecological Data Using CANOCO. Cambridge University Press.

Miserendino ML, Pizzolon LA. 2003. Distribution of macroinvertebrate assemblages in the Azul-Quemquemtreu river basin, Patagonia, Argentina. New Zeal. J. Mar. Fresh., 37: 525-539.

Morais MM. 1995. Organização especial e temporal de um rio temporário mediterrânico (Rio Degebe, bacia hidrográfica do Guadiana). Descritores fisico-químicos e produtores primarios. Comunidades bentónicas de macroinvertebrados. PhD Thesis, University of Évora, Évora, Portugal.

MOULAY DEJ. 2005. Croissance urbaine et problèmes d'assainissement liquide et pluvial dans le bassin du Srou (Maroc central). Sécheresse, 16: 41-52

Muli JR, Mavuti KM. 2001. The benthic macro fauna community of Kenyan waters of Lake Victoria. Hydrobiologia, 458: 83-90.

Ndaruga AM, Ndiritu GG, Gichuki NN, Wamicha WN. 2004. Impact of water quality on macroinvertebrate assemblages along a tropical stream in Kenya. Afr. $J$. Ecol., 42: 208-216.

Nedeau EJ, Merritt RW, Kaufman MG. 2003. The effect of an industrial effluent on an urban stream benthic community: water quality vs habitat quality. Environ Pollut., 123(1): 1-13.

Negishi Richardson. 2003. Responses of organic matter and macroinvertebrates to placements of boulder clusters in a small stream of southwestern British Columbia, Canada. Can. J. Fish. Aquat. Sci., 60: 247-258.

Nwokedi GIC, Obodo GA, Nwankwo SI. 1992. Pollution chemistry of the river Niger and its tributaries: characteristics of industrial waste effluents. Bull. Environ. Con. and Tox., 48: 441-448.

Odum EP. 1972. Fundamentals of Ecology. W. A. Soundes Company: Philadelphia; $574 \mathrm{p}$.

Ogbogu SS. 2001. Factors affecting the distribution and abundance of Cloen and Caenis (Ephemeroptera) larvae in a tropical impounded river, Nigeria. Afr. J. Ecol., 39: 106-112.

Ometo JPHB, Martinelli LA, Ballester MV, Gessner A, Krusche AV, Victoria RL, Williams M. 2000. Effects of land use on water chemistry and macroinvertebrates in two streams of the Piracicaba river basin, Southeast Brazil. Freshw. Biol., 44: 327-337.

Picouet C. 1999. Géodynamique d'un hydrosystème tropical peu anthropisé : le bassin supérieur du Niger et son delta 
intérieur. Thèse de Doctorat, Univ. Montpellier II, $469 \mathrm{p}$.

Piscart C, Moreteau JC, Beisel JN. 2005. Biodiversity and structure of macroinvertebrate communities along a small permanent salinity gradient (Meurthe River, France). Hydrobiologia, 551: 227-236.

Piyankarage SC, Mallawatantri AP, Matsuno Y, Pathiratne KAS. 2004. Human impacts and the status of water quality in the Bundala RAMSAR wetland lagoon system in Southern Sri Lanka. Wetlands Ecol. Manag., 12: 473-482.

Plea M, Cissé AS, Keïta K, Doumbia A. 2005. Qualité des eaux du fleuve Niger à Bamako: impacts des activités domestiques. Conférence débat sur la problématique de l'eau au Mali. Univ. Bamako, présentation orale.

Quinn JM, Hickey CW. 1990. Magnitude of effects of substrate particle size, recent flooding, and catchment development on benthic invertebrates in 88 New Zealand rivers. In Characterisation and classification of benthic invertebrate communities in 88 New Zealand rivers in relation to environmental factors. New Zeal. J. Mar. Fresh, 24: 387-409.

Richards C, Host GE, Arthur JW. 1993. Identification of predominant environmental factors structuring stream macroinvertebrate communities within a large agricultural catchment. Freshw. Biol., 29: 285-294.

Roy AH, Rosemond AD, Paul MJ, Leigh DS, Wallage JB. 2003. Stream macroinvertebrate response to catchment urbanisation (Georgia, U.S.A). Freshw. Biol., 48: 329-346.

Sarkar SK, Bhattacharya A, Giri S, Bhattacharya B, Sarkar D, Nayak DC,
Chattopadhaya AK. 2005. Spatiotemporal variation in benthic polychaetes (Annelida) and relationships with environnemental variables in a tropical estuary. Wetlands Ecol. Manag., 13: 5567.

Shivoga WA. 2001. The influence of hydrology on the structure of invertebrate communities in two streams flowing into Lake Nakuru, Kenya. Hydrobiologia, 458: 121-130.

Spellerberg IF, Fedor PJ. 2003. A tribute to Claude Shannon (1916-2001) and a plea for more rigorous use of species richness, species diversity and the 'ShannonWiener' index. Global Ecol. Biogeogr., 12: 177-179.

Statsoft France. 1997. Statistica pour Windows (manuel du programme): Statsoft France - 31, cours des Juilliottes - 94700 Maisons - Alfort.

Statzner B, Higler B. 1986. Stream hydraulics as a major determinant of benthic invertebrate zonation patterns. Freshwater Biol., 16: 127-139.

Tarr TL, Baber MJ, Babbitt KJ. 2005. Macroinvertebrate community structure across a wetland hydroperiod gradient in southern New Hampshire, USA. Wetlands Ecol. Manag., 13: 321-334.

Ter Braak CJF, Smilauer P. 1999. CANOCO for Windows (version 4.02) - a FORTRAN program for canonical community ordination. Centre for biometry Wageningen. Wageningen. The Netherlands.

Uzoukwu AB, Ngoka C, Nneji N. 2004. Monitoring of seasonal variation in the water quality of Ubu river in Ekwusigo and Nnewi local government areas of Anambra State, Nigeria. Environ. Manage., 33: 886-898. 